\title{
Revealing the Compact Structure of Lactic Acid Bacterial Heteroexopolysaccharides by SAXS and DLS
}

Khan, Sanaullah; Birch, Johnny; Harris, Pernille; Van Calsteren, Marie-Rose; Ipsen, Richard; Peters, Günther H.J.; Svensson, Birte; Almdal, Kristoffer

\author{
Published in: \\ Biomacromolecules \\ Link to article, DOI: \\ 10.1021/acs.biomac.6b01597 \\ 10.1021/acs.biomac.6601597
}

Publication date:

2017

Document Version

Peer reviewed version

Link back to DTU Orbit

Citation (APA):

Khan, S., Birch, J., Harris, P., Van Calsteren, M-R., Ipsen, R., Peters, G. H. J., Svensson, B., \& Almdal, K. (2017). Revealing the Compact Structure of Lactic Acid Bacterial Heteroexopolysaccharides by SAXS and DLS. Biomacromolecules, 18(3), 747-757. https://doi.org/10.1021/acs.biomac.6b01597, https://doi.org/10.1021/acs.biomac.6601597

\section{General rights}

Copyright and moral rights for the publications made accessible in the public portal are retained by the authors and/or other copyright owners and it is a condition of accessing publications that users recognise and abide by the legal requirements associated with these rights.

- Users may download and print one copy of any publication from the public portal for the purpose of private study or research.

- You may not further distribute the material or use it for any profit-making activity or commercial gain

- You may freely distribute the URL identifying the publication in the public portal 


\section{Revealing the compact structure of lactic acid bacterial}

\section{hetero-exopolysaccharides by SAXS and DLS}

Sanaullah Khan ${ }^{\dagger}$, Johnny Birch ${ }^{\ddagger}$, Pernille Harris ${ }^{\S}$, Marie-Rose Van Calsteren ${ }^{\Perp}$, Richard Ipsen ${ }^{\perp}$, Günther H.J. Peters ${ }^{\S}$, Birte Svensson ${ }^{\ddagger}$ and Kristoffer Almdal ${ }^{\dagger *}$

${ }^{\dagger}$ Department of Micro- and Nanotechnology, DTU, Ørsteds Plads, building 423, DK-2800 Kgs. Lyngby, Denmark.

${ }^{\ddagger}$ Enzyme and Protein Chemistry, Department of Systems Biology, DTU, Elektrovej, building 375, DK2800 Kgs. Lyngby, Denmark.

${ }^{\S}$ Department of Chemistry, DTU, Kemitorvet, building 207, DK-2800 Kgs. Lyngby, Denmark

"Saint-Hyacinthe Research and Development Centre, Agriculture and Agri-Food Canada, 3600 Casavant Boulevard West, Saint-Hyacinthe, Quebec J2S 8E3, Canada.

${ }^{\perp}$ Department of Food Science, University of Copenhagen, Rolighedsvej 26, DK-1958 Frederiksberg Denmark 


\section{ABSTRACT}

Molecular structures of exopolysaccharides are required to understand their functions and the relationships between the structure and physical and rheological properties. Small-angle X-ray scattering and dynamic light scattering were used in conjunction with molecular modeling to characterize solution structures of three lactic acid bacterial hetero-exopolysaccharides (HePS-1, HePS2 and HePS-3). Values of radius of gyration $R_{\mathrm{G}}$, cross-sectional radius of gyration $R_{\mathrm{XS}}$, approximate length $L$ and hydrodynamic diameter were not directly proportional to the molar mass and indicated the HePSs adopted a compact coil-like rather than an extended conformation. Constrained molecular modeling of 15,000 randomised HePS-1 conformers resulted in five best-fit structures with $R$ factor of 3.9-4.6\% revealing random coil-like structure. $\Phi$ and $\Psi$ angle analysis of glycosidic linkages in HePS-1 structures suggests Galf residues significantly influence the conformation. Ab initio scattering modeling of HePS-2 and HePS-3 gave excellent curve fittings with $\chi^{2}$ of 0.43 and 0.34 for best-fit models, respectively, compatible with coil-like conformation. The findings disclose solution behaviour of HePS relevant for their interactions with biomacromolecules e.g. milk proteins.

Keywords: Hetero-exopolysaccharides; small-angle X-ray scattering; constrained scattering modeling; dynamic light scattering 


\section{INTRODUCTION}

Exopolysaccharides (EPSs) are biopolymers secreted by a vast number of microorganisms including yeasts, molds, microalgae and bacteria. ${ }^{1,2}$ EPSs have wide diversity in monosaccharide composition and glycosidic linkages of their oligosaccharide repeat units. ${ }^{3}$ Bacterial EPSs are grouped into two classes, namely homoexopolysaccharides (HoPSs) and heteroexopolysaccharides (HePSs). HoPSs contain a single type of monosaccharide units (mostly D-glucopyranose or D-fructofuranose) and are synthesized from sucrose by glucansucrases and fructansucrases, respectively. ${ }^{4}$ HePSs by contrast are composed of more than one type of monosaccharide in oligosaccharide repeats of 3 to 9 residues synthesized from intracellular sugar nucleotide precursors and then polymerized. ${ }^{5-8}$ Typical lactic acid bacteria (LAB) HoPS and HePS contain a linear polysaccharide backbone with branches of varying lengths.

The physiological function of EPSs is not fully known. Generally, in their natural environment EPSs are believed to protect microbial cells against desiccation, phagocytosis, antibiotics, environmental stress and are involved in adhesion to solid surfaces, biofilm formation and cell recognition. ${ }^{7-10}$ EPSs receive considerable attention in various food and pharmaceutical industries due to their technological properties and GRAS ("generally regarded as safe") status. ${ }^{11,12}$ In the food industry, LAB-EPSs serve as viscosifying, emulsifying and gelling agents to improve rheological and textural properties of food products. $^{9,} 12,13$ Moreover, LAB-EPSs have also been described to exert health benefits, e.g. lowering cholesterol levels, ${ }^{10,14,15}$ reducing formation of biofilms, ${ }^{16}$ modulating adhesion to epithelial cells and inducing a prebiotic effect by increasing bifidobacteria levels in the intestinal tract. ${ }^{17,18}$

The efficacious applications of EPSs are considered to result from their capability to regulate viscosity and bind water, which correlate to their structure and interactions with proteins. ${ }^{17,19,20}$ The 
molecular weight of EPSs is one of several factors contributing to their functional properties in solution. ${ }^{21}$ Other factors including the three-dimensional (3D) structure and conformational flexibility connected with degree of branching, ${ }^{22,23}$ charge,$^{24,25}$ and ability to engage in intermolecular association significantly influence physical and rheological properties of EPSs. ${ }^{10}$ The nature of glycosidic linkages and sugar ring configuration also impacts viscosity. High viscosities are associated with high molar mass, chain stiffness and extended structures of EPSs which tend to occupy larger volumes in solution. $^{21,26}$ The degree of chain stiffness is related to the presence of glycosidic linkages with less rotational freedom in the EPS structure. For example $\beta-(1 \rightarrow 4)$ linkages are stiffer than $\beta-(1 \rightarrow 3)$ and $\beta$ $(1 \rightarrow 2)$ glycosidic linkages, hence contributing to the viscosity. ${ }^{27}$

To expand knowledge on EPS conformation in solution and the relationship to physical and rheological properties, experimental analysis is needed of the molecular structures of intact EPSs. High resolution X-ray crystallography and nuclear magnetic resonance (NMR) methods that are widely used to obtain 3D structures of biomacromolecules are not suited for EPS structure determination as X-ray crystallography requires well ordered crystals of good diffraction quality, and NMR is limited to comparatively small macromolecules. The extreme flexibility, large size, high viscosity and modest water solubility of EPSs precluded their 3D structure determination to date. In the present study, we characterize three HePSs (HePS-1, HePS-2 and HePS-3) from Streptococcus thermophilus ${ }^{28}$, Lactobacillus rhamnosus $^{29}$ and Lactobacillus delbrueckii ssp. bulgaricus ${ }^{30}$ using small-angle X-ray scattering (SAXS) and Dynamic Light Scattering (DLS). The solution scattering in conjunction with constrained scattering modeling was used to determine the molecular structure of HePS- 1 . This approach is well-established and has been used for solution structure determination of multi-domain proteins and anionic oligosaccharides, ${ }^{31-34}$ but has not previously been used for structural analysis of 
large intact EPSs. The data provide new insight into structural features of HePSs and form a basis for identifying structural requirements enabling binding to biomacromolecules such as milk proteins.

\section{MATERIALS AND METHODS}

Production, purification and quantification of HePSs. HePS-1, HePS-2 and HePS-3 were produced in $10 \%$ skimmed milk medium (10\% skimmed milk powder in MilliQ (MQ) water) by Streptococcus thermophilus EU20 (kind gift of Prof. Luc De Vuyst, Vrije Universiteit Brussels, Belgium), ${ }^{28}$ Lactobacillus rhamnosus GG (ATCC 53103) ${ }^{29}$ and Lactobacillus delbrueckii ssp. bulgaricus (NCIMB 702483) ${ }^{30}$, respectively.

HePS-1 was isolated from the culture medium as described previously. ${ }^{35}$ Briefly, proteins and cells were precipitated by addition of trichloroacetic acid (TCA) to a final concentration of $20 \%$ and removed by centrifugation $\left(15,000 \mathrm{~g}, 30 \mathrm{~min}, 4^{\circ} \mathrm{C}\right)$. HePS- 1 was precipitated overnight $\left(5^{\circ} \mathrm{C}\right)$ from the supernatant by adding ethanol $(2: 1, \mathrm{v} / \mathrm{v})$, isolated by centrifugation as above, redissolved in MQ water $\left(1 \mathrm{~h}, 50^{\circ} \mathrm{C}\right)$ and residual proteins were precipitated by TCA followed by ethanol-precipitation of HePS1 (both steps as above). HePS-1 was dissolved in MQ-water as above, dialyzed (50,000 kDa cutoff, $20^{\circ} \mathrm{C}$ ) against MQ water (5 d, water replacement twice a day) and finally purified by size-exclusion chromatography (XK26/100 Sephacryl S-400; GE Healthcare, Uppsala, Sweden) and lyophilized. HePS-2 and HePS-3 were purified from cultures by TCA precipitation (final concentration 20\% (w/v)) of cells and proteins for $20 \mathrm{~min}$ at room temperature, centrifugation (as above), followed by the HePS precipitation overnight by adding acetone $\left(1: 1 \mathrm{v} / \mathrm{v} ; 4^{\circ} \mathrm{C}\right)$, centrifugation $\left(12,000 \mathrm{~g}, 30 \mathrm{~min}, 4^{\circ} \mathrm{C}\right)$, redissolution in MQ water, extraction by 1:1 (v/v) phenol/chloroform/isoamyl alcohol 25:24:1 twice and extraction by 1:1 (v/v) of chloroform/isoamyl alcohol 24:1 once 
. Following acetone precipitation and centrifugation (as above), the purified HePS was dissolved in deionized water, dialyzed, filtered and freeze-dried.

HePS concentrations were quantified using the phenol-sulfuric acid method and monosaccharide mixtures corresponding to the composition of the various repeat units as standards. ${ }^{36}$

Molecular weight $\left(\boldsymbol{M}_{\mathbf{w}}\right)$ Determination. Molecular weights of HePS-1- HePS-3 were determined by size exclusion chromatography (SEC). The SEC system consists of a solvent delivery system (LC10AD), autosampler (SIL-10A), RI detector (RID-10A; all Shimadzu, Kyoto, Japan) and a size exclusion column (OH-PK SB-805 HQ $300 \times 8 \mathrm{~mm}$, Tokyo, Japan). Dextran standards of $M_{\mathrm{w}} 4.5 \mathrm{MDa}$, 1.45 MDa, $560 \mathrm{kDa}, 350 \mathrm{kDa}$ (American Polymer Standards Corporation, Mentor, Ohio, USA), 276.5 kDa, 196.3 kDa, 123 kDa, 43 kDa (Pharmacosmos, Holbaek, Denmark) and pullulan of $22 \mathrm{kDa}$ were used for calibration. Standards $\left(1-2.7 \mathrm{mg} \mathrm{ml}^{-1}\right)$ and HePSs $\left(1-2 \mathrm{mg} \mathrm{ml}^{-1}\right)$ were dissolved in mobile phase (10 mM sodium citrate/citric acid $\mathrm{pH} 4.0)$, degassed, kept overnight, filtered $(0.45 \mu \mathrm{m}$ filters; Frisenette ApS, Knebel, Denmark) and $100 \mu 1$ was subjected to SEC at a flow rate of $0.5 \mathrm{ml} \mathrm{min}{ }^{-1}$.

NMR characterization of HePS-3. HePS-3 $(\sim 10 \mathrm{mg} / \mathrm{mL})$ was exchanged in $\mathrm{D}_{2} \mathrm{O}$, and the NMR spectrum was obtained on a Chemagnetics (Fort Collins, CO, USA) CMX Infinity 300 spectrometer equipped with a $5-\mathrm{mm}$ dual ${ }^{13} \mathrm{C} /{ }^{1} \mathrm{H}$ Nalorac (Martinez, CA, USA) probe. The temperature was controlled at $70^{\circ} \mathrm{C}$. The ${ }^{1} \mathrm{H}$ NMR experiment was performed with original pulse program of the Spinsight software. For the 1D spectrum, $8 \mathrm{~K}$ complex data points were acquired and processed by exponential multiplication with a line broadening factor equal to the digital resolution, complex Fourier transform, phase correction and third-order polynomial baseline correction.

Small angle X-ray scattering (SAXS). Experiments with HePS-1, HePS-2 and HePS-3 were performed on beamline I911-4 at the MAX IV (synchrotron radiation facility, Lund Sweden). ${ }^{37}$ The 
sample detector distance and the direct beam position were calibrated using silver behenate. Pure water was used to place the data on an absolute scale. Buffers were measured before each sample and averaged before subtraction. The sample size was approximately $40 \mu \mathrm{L}$ using an automated flow cell. Eight time frames were measured using $20 \mathrm{~s}$ exposure time optimized using on-line checks during acquisitions to avoid radiation damage effects. The frames were averaged to maximize signal-to-noise ratios. Initial data treatment was done using the in-house software Bli911-4. Buffer averaging and subtraction prior to data analysis was done in Primus. ${ }^{38}$ Data were collected for all three HePSs at concentrations of $1-2 \mathrm{mg} / \mathrm{mL}$ in $10 \mathrm{mM}$ sodium citrate, $\mathrm{pH} 4.0$. Guinier analysis gives the radius of gyration $R_{\mathrm{G}}$, which measures the degree of structural elongation in solution if the internal inhomogeneity of scattering within the macromolecules has no effect. Guinier plots at low $Q$ (where $Q$ $=4 \pi \sin \theta / \lambda ; 2 \theta$ is the scattering angle and $\lambda$ is the wavelength) give $R_{\mathrm{G}}$ and the forward scattering at zero angle $I(0):{ }^{39}$

$$
\ln I(Q)=\ln I(0)-R_{\mathrm{G}}^{2} Q^{2} / 3
$$

If the structure is elongated (i.e. rod-shaped), the radius of gyration of the cross-sectional structure $R_{\mathrm{XS}}$ and the mean cross-sectional intensity at zero angle $[I(Q) \cdot Q]_{Q \rightarrow 0}$ parameters are obtained from:

$$
\ln [I(Q) \cdot Q]=\ln [I(Q) \cdot Q]_{Q \rightarrow 0}-R_{\mathrm{XS}}^{2} Q^{2} / 2
$$

The $R_{\mathrm{G}}$ and $R_{\mathrm{XS}}$ analyses were done using PRIMUS. ${ }^{38}$ Indirect Fourier transformation of the full scattering curve $I(Q)$ in reciprocal space gives the distance distribution function $P(r)$ in real space. This yields the maximum dimension of the macromolecule $L$ and its most commonly occurring distance vector $M$ in real space: 


$$
P(r)=\frac{1}{2 \pi^{2}} \int_{0}^{\infty} I(Q) Q r \sin (Q r) \mathrm{d} Q
$$

The transformation was carried out using GNOM software. ${ }^{40}$

Molecular modeling of HePS-1. 3D models of HePS-1-HePS-3 repeating units were built using the Sweet 2 web server (sweet2) ${ }^{41,42}$ from their structures determined by NMR (Figure 1). The $\Phi$ and $\Psi$ angle values in HePS-1 repeating unit are shown in Table 2. A fully extended HePS-1 structure was generated by joining 128 repeat units through glycosidic linkages using Discovery Studio (version 4.5) molecular graphics software (Accelrys, San Diego, CA). Fully extended models for HePS-2 and HePS3 were also generated by joining about 389 and 206 repeating units, respectively using Discovery Studio. These models were generated in accordance with their molecular weights determined by size exclusion chromatography.

A total of 15,000 conformationally randomized models for HePS-1 were generated from the extended (linear) model using the TorsionKick function in a PERL script modified from the ExtractAngle.pl script provided with the Discovery Studio software. ${ }^{33,34}$ The TorsionKick takes any value in a maximum range of $\pm 45^{\circ}$ from their starting values. A constant force field termed DREIDING minimization was used to avoid steric clashes between the atoms in each randomized structure. $^{43}$

Constrained scattering modeling of HePS-1. X-ray scattering curves were calculated for each randomized HePS-1 model for comparison with the experimental curves using the SCT program. ${ }^{44,45}$ A cube side length of $0.527 \mathrm{~nm}$ and a cutoff of 4 atoms were used to create spheres in the HePS-1 models. The hydration shell $\left(0.3 \mathrm{~g} \mathrm{H}_{2} \mathrm{O} / \mathrm{g}\right.$ protein $)$ was created by adding spheres to the unhydrated sphere 
models using HYPRO. ${ }^{45,46}$ The X-ray scattering curve $I(Q)$ was calculated using the Debye equation as adapted to spheres and assuming a uniform scattering density for the spheres: ${ }^{44,47}$

$$
\begin{aligned}
& \frac{I(Q)}{I(0)}=g(Q)\left(n^{-1}+2 n^{-2} \sum_{j=1}^{m} A j \frac{\sin Q r_{j}}{Q r_{j}}\right) \\
& g(Q)=(3(\sin Q R-Q R \cos Q R))^{2} / Q^{6} R^{6}
\end{aligned}
$$

where $g(Q)$ is the squared form factor for the sphere of radius $r, n$ is the number of spheres filling the body, $A_{j}$ is the number of distances $r_{j}$ for that value of $j, r_{j}$ is the distance between the spheres, and $m$ is the number of different distances $r_{j}$. The models were assessed by calculation of the radius of gyration $R_{\mathrm{G}}$ and cross-sectional $R_{\mathrm{XS}}$ values in the same $Q$ ranges used for the experimental Guinier fits. Models that were filtered using the $R_{\mathrm{G}}$ and $R_{\mathrm{Xs}}$ filters were then ranked using a goodness-of-fit $R$ factor in order to identify the best-fit models.

$\boldsymbol{A} \boldsymbol{b}$ initio scattering modeling of HePS-2 and HePS-3. SAXS data of HePS-2 and HePS-3 were used to derive $a b$ initio information on their shapes and conformation. To generate a molecular envelope of HePS-2 and HePS-3 from scattering data, $20 a b$ initio beads models were generated for each HePS using DAMMIF program, ${ }^{48}$ assuming no P1 symmetry. These models were filtered and averaged in DAMAVER ${ }^{47}$ in the ATSAS package. ${ }^{50}$

Dynamic light scattering (DLS). Particle size distribution data for HePS-1, HePS-2 and HePS-3 were obtained at a scattering angle of $90^{\circ}$ (BI-200SM instrument; Brookhaven Instruments Corporation; Holtsville, NY, USA) and $23^{\circ} \mathrm{C}$. DLS analyses the size distribution of particles undergoing Brownian motion through illumination by a laser beam. The time-dependent fluctuations in the intensity of scattered light reflect the rate at which the particles diffuse. The distributions of mean apparent translational diffusion coefficients $\left(D_{\mathrm{T}}\right)$ were determined by fitting the DLS autocorrelation 
functions obtained by the Brookhaven system using non-negative constrained least-squares (NNLS). The distribution of $D_{\mathrm{T}}$ values was converted to the distribution of hydrodynamic diameters $\left(d_{\mathrm{H}}\right)$ using the Stokes-Einstein equation:

$$
d_{\mathrm{H}}=k T / 3 \pi \eta D_{\mathrm{T}}
$$

where $\mathrm{k}$ is the Boltzmann constant, $\mathrm{T}$ the absolute temperature, and $\eta$ the solvent viscosity (assumed to be that of water, $0.933 \mathrm{mPa} \cdot \mathrm{s})$.

\section{RESULTS AND DISCUSSION}

HePSs repeat units and molecular weight $\left(\boldsymbol{M}_{\mathbf{w}}\right)$. Primary structures of HePS-1, HePS-2 and HePS3 consist of a branched repeat units of $\mathrm{dp} 7$ (where dp stands for degree of polymerization) composed of glucose, rhamnose and galactose (molar ratio of 2:2:3), ${ }^{28}$ dp6 of $\mathrm{N}$-acetyl-glucosamine, rhamnose and galactose (molar ratio of $1: 1: 4),{ }^{29} \mathrm{dp} 7$ of glucose, rhamnose and galactose (molar ratio of 1:1:5) as shown in Figure 1. Analysis of the ${ }^{1} \mathrm{H}$ NMR spectrum of HePS-3 (data not shown) revealed that the HePS-3 repeating unit structure is identical to that previously described for the HePS purified from Lactobacillus delbrückii subspecies bulgaricus strain $\mathrm{rr}^{35}$ The $M_{\mathrm{w}}$ was determined to be $145 \pm 4 \mathrm{kDa}$ for HePS-1, $389 \pm 6 \mathrm{kDa}$ for HePS-2 and $372 \pm 8 \mathrm{kDa}$ for HePS-3 by SEC (data not shown). 
$\begin{array}{cc} & \alpha-\mathrm{L}-\mathrm{Rh} \text { ap } \\ & 1 \\ & \downarrow \\ \text { ePS-1 } & 2\end{array}$

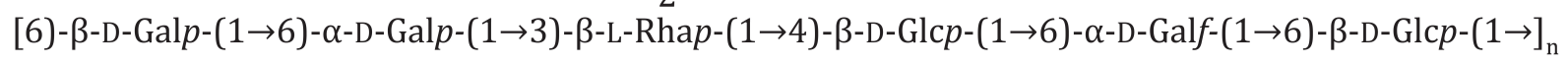

HePS-2

$$
\begin{gathered}
\beta \text {-D-Gal } f \\
1 \\
\downarrow
\end{gathered}
$$

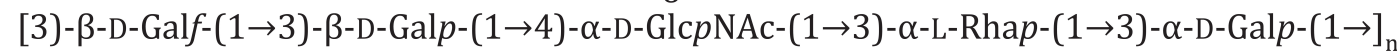

HePS-3

$\begin{array}{ccc}\beta \text {-D-Gal } p & \beta \text {-D-Gal } p & \alpha \text {-L-Rhap } \\ 1 & 1 & 1 \\ \downarrow & \downarrow & \downarrow \\ 3 & 4 & 3\end{array}$

[2)- $\alpha$-D-Gal $p-(1 \rightarrow 3)-\beta-D-G l c p-(1 \rightarrow 3)-\beta-D-G a l p-(1 \rightarrow 4)-\alpha-D-G a l p-(1 \rightarrow]_{\mathrm{n}}$

Figure 1. Primary structures of HePS-1, HePS-2 andHePS-3 repeating units. HePS-1 was purified from Streptococcus thermophilus EU20, HePS-2 from Lactobacillus rhamnosus GG (ATCC 53103) and HePS-3 from Lactobacillus delbrueckii ssp. bulgaricus (NCIMB 702483).

SAXS of HePS-1, HePS-2 andHePS-3. HePS-1,HePS-2 and HePS-3 (1-2 mg/mL) were characterized by SAXS to gain insight into their overall structure in solution. ${ }^{31}$ The Guinier analyses of $\ln I(Q)$ versus $Q^{2}$ at low $Q$ values give the radius of gyration $R_{\mathrm{G}}$ that monitors the extent of macromolecular elongation. The Guinier fits resulted in an $R_{\mathrm{G}}$ value of $10.14 \pm 0.31 \mathrm{~nm}$ for HePS-1 using a $Q$ range of $0.1-0.15 \mathrm{~nm}^{-1}$, one of $11.72 \pm 0.23 \mathrm{~nm}$ for HePS-2 using a $Q$ range of $0.09-0.12 \mathrm{~nm}^{-1}$ and one of $10.82 \pm 0.48 \mathrm{~nm}$ for HePS-3 using a $Q$ range of $0.09-0.13 \mathrm{~nm}^{-1}$ (Figure 2A, Table 1). The experimental $R_{\mathrm{G}}$ values do not show a linear relationship with modeled $R_{\mathrm{G}}$ values of $91 \mathrm{~nm}, 230 \mathrm{~nm}$ and $139 \mathrm{~nm}$ calculated for extended chain lengths of HePS-1, HePS-2 and HePS3, respectively, indicating that the HePSs are not completely extended rods but adopt coil-like conformations in solution.

For elongated macromolecules, the cross-sectional radius of gyration $\left(R_{\mathrm{Xs}}\right)$ provides information on the degree of bending within the molecular length. The $R_{\mathrm{Xs}}$ analyses of 
$\ln I(Q) Q$ versus $Q^{2}$ showed good fits for HePS-1, HePS-2 and HePS-3 in a $Q$ range of $0.18-0.31$ $\mathrm{nm}^{-1}, 0.18-0.27 \mathrm{~nm}^{-1}$ and $0.17-0.27 \mathrm{~nm}^{-1}$ respectively, resulting in experimental $R_{\mathrm{Xs}}$ values of $3.22 \pm 0.09 \mathrm{~nm}$ for HePS-1, $3.95 \pm 0.01 \mathrm{~nm}$ for HePS-2 and $3.50 \pm 0.09 \mathrm{~nm}$ for HePS-3 (Figure 2B, Table 1). If the HePSs are approximated as elliptical cylinders, the combination of $R_{\mathrm{G}}$ and $R_{\mathrm{Xs}}$ according to the relationship $L^{2}=12\left(R_{\mathrm{G}}{ }^{2}-R_{\mathrm{XS}}{ }^{2}\right)$ resulted in experimental approximate length $L$ of 33.3, 38.2 and $35.5 \mathrm{~nm}$ for HePS-1, HePS-2 and HePS-3, respectively.

The distance distribution function $P(r)$ represents the distribution of all the distances between the atoms within the macromolecule. It provides $R_{\mathrm{G}}$ values and model-independent determinations of overall lengths, $L$. The mean $R_{\mathrm{G}}$ obtained from the $P(r)$ curves were $10.89 \pm 0.16$ $\mathrm{nm}, 11.49 \pm 0.25 \mathrm{~nm}$ and $11.44 \pm 0.1 \mathrm{~nm}$ for HePS-1, HePS-2 and HePS-3, respectively (Table 1), which agree with the values obtained from the corresponding Guinier $R_{\mathrm{G}}$ analyses.
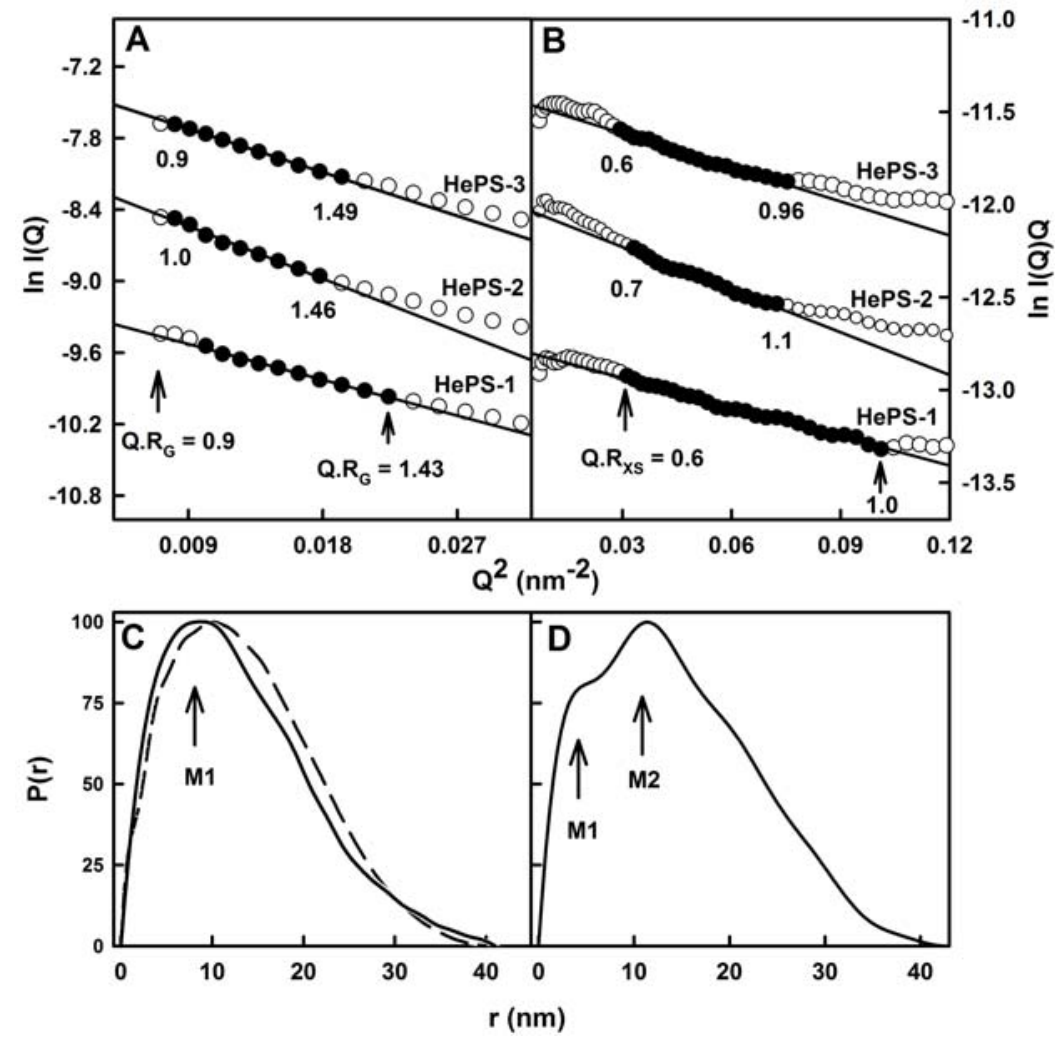
Figure 2. Experimental Guinier $R_{\mathrm{G}}, R_{\mathrm{XS}}$ and $P(r)$ analyses of HePS-1, HePS-2 and HePS-3. (A) Guinier $R_{\mathrm{G}}$ plots for HePS-1 at $1 \mathrm{mg} / \mathrm{ml}$, and HePS-2 and HePS-3 at $2.0 \mathrm{mg} / \mathrm{ml}$. Filled circles represent the $Q$ ranges used to determine the radius of gyration $R_{\mathrm{G}}$ based on the best-fit lines as shown. The $Q$ ranges used for $R_{\mathrm{G}}$ analyses were $0.1-0.15 \mathrm{~nm}^{-1}$ for HePS-1, 0.09-0.12 $\mathrm{nm}^{-1}$ for HePS-2 and $0.09-0.13 \mathrm{~nm}^{-1}$ for HePS-3. (B) Guinier cross-sectional $R_{\mathrm{Xs}}$ plots for HePS-1, HePS-2 and HePS-3. The filled circles represent the $Q$ ranges used to determine the $R_{\mathrm{XS}}$ values based on the best-fit lines as shown. The $Q$ ranges used for $R_{\mathrm{XS}}$ analyses were $0.18-0.31 \mathrm{~nm}^{-1}$ for HePS-1, 0.18 $0.27 \mathrm{~nm}^{-1}$ for HePS-2 and $0.17-0.27 \mathrm{~nm}^{-1}$ for HePS-3. (C) Normalized $P(r)$ curve for HePS1-HePS-2. The M1 value of $8.7 \mathrm{~nm}$ and $L$ value of $41.0 \mathrm{~nm}$ were observed for HePS-1 (solid line). The M1 value of $9.9 \mathrm{~nm}$ and $L$ value of $38.0 \mathrm{~nm}$ were observed for HePS-2 (broken line). (D) Normalized $P(r)$ curve for HePS-3. The $r$ values of the maximum at M1 and M2 were $3.9 \mathrm{~nm}$ and $11.3 \mathrm{~nm}$, respectively, and the maximum length $L$ values as 41.8 .

Values of the maximum length $L$ were determined from the $r$ value where the $P(r)$ curve reaches zero at large $r$. The experimental $L$ values were $41.0 \mathrm{~nm}, 38.0 \mathrm{~nm}$ and $41.8 \mathrm{~nm}$ for HePS-1, HePS-2 and HePS-3, respectively (Figure 2C and D). Although the three HePSs differ in molar mass, their lengths 
Table 1. SAXS and scattering modeling fits for the solution structures of HePSs

\begin{tabular}{|c|c|c|c|c|c|c|c|c|}
\hline HePSs & Filter & $\begin{array}{l}\text { Number of } \\
\text { models }\end{array}$ & $R_{\mathrm{G}}(\mathrm{nm})^{\mathrm{a}}$ & $R_{\mathrm{XS}}(\mathrm{nm})$ & $\begin{array}{l}R \text { factor } \\
(\%)\end{array}$ & $\chi^{2}$ & $\begin{array}{l}\text { Length } L \\
(\mathrm{~nm})\end{array}$ & $d_{\mathrm{H}}(\mathrm{nm})$ \\
\hline \multirow[t]{5}{*}{ HePS-1 } & None & 15,000 & $2.80-17.80$ & $0.04-7.9$ & $3.9-32.8$ & & n.a & \\
\hline & $R_{\mathrm{G}}, R_{\mathrm{XS}}, R$ factor & 5 & $9.90-10.84$ & $3.14-3.47$ & $3.9-4.6$ & & $42-44$ & \\
\hline & Best-fit & 1 & 10.35 & 3.33 & 3.9 & & 42.0 & \\
\hline & Experimental & & $10.14 \pm 0.31$ & $3.22 \pm 0.09$ & & & 41.0 & $29.9 \pm 0.2$ \\
\hline & & & $10.89 \pm 0.16$ & & & & & \\
\hline \multirow[t]{3}{*}{ HePS-2 } & Ab initio models & 20 & $11.50-11.61$ & & & $0.43-0.50$ & $37-42$ & \\
\hline & Best-fit & 1 & 11.56 & & & 0.43 & 39.6 & \\
\hline & Experimental & & $\begin{array}{l}11.72 \pm 0.23 \\
11.49 \pm 0.25\end{array}$ & $3.95 \pm 0.01$ & & & 38.0 & $34.1 \pm 0.3$ \\
\hline \multirow[t]{3}{*}{ HePS-3 } & Ab initio models & 20 & $11.45-11.63$ & & & $0.34-0.40$ & $35.6-41.0$ & \\
\hline & Best-fit & 1 & 11.63 & & & 0.34 & 39.0 & \\
\hline & Experimental & & $\begin{array}{l}10.82 \pm 0.48 \\
11.44 \pm 0.1\end{array}$ & $3.50 \pm 0.09$ & & & 41.8 & $35.6 \pm 0.5$ \\
\hline
\end{tabular}

${ }^{\mathrm{a}}$ The first value is from the Guinier $R_{\mathrm{G}}$ analyses, and the second from the GNOM $P(r)$ analyses. 
are very close each other, showing that the HePSs of higher molar mass are comparatively more compact than the one of lower molar mass. These $L$ values are much smaller than those calculated assuming that the HePS-1, HePS-2 and HePS-3 models have maximally extended molecular conformations of lengths $321 \mathrm{~nm}, 790 \mathrm{~nm}$ and $475 \mathrm{~nm}$, respectively. This large difference indicated that the HePSs do not have extended (linear) backbone conformations in solution, being compact instead. The approximate $L$ values of HePS-1 and HePS-3 estimated from $R_{\mathrm{G}}$ and $\mathrm{R}_{\mathrm{XS}}$ values were comparable to those from the $P(r)$ curves. The $L$ values of HePS-2 from $R_{\mathrm{G}}$ and $\mathrm{R}_{\mathrm{XS}}$ values and the $P(r)$ curves were in similar range. It is important to mention that these experimental $L$ values from the $P(r)$ curves are more accurate than the $L$ values determined from the $R_{\mathrm{G}}$ and $R_{\mathrm{XS}}$ analyses and the hydrodynamic diameters measured by DLS (Table 1). Even so, the DLS data and these approximate lengths show that $R_{\mathrm{G}}$ and $R_{\mathrm{XS}}$ values are consistent with the $P(r)$ analyses. The maximum $\mathrm{M}$ in $P(r)$ curves corresponds to the most frequently occurring interatomic distance within the HePSs. For HePS-1 and HePS-2, one peak was observed at $r$ values of $8.7 \mathrm{~nm}$ and $9.9 \mathrm{~nm}$ respectively (Figure 2C), while for HePS-3, two maxima M1 and M2 were observed at $r$ values of $3.9 \mathrm{~nm}$ and $11.3 \mathrm{~nm}$ respectively (Figure $2 \mathrm{D})$.

The HePSs have many similarities to synthetic polymers. The isolated HePS-1, HePS-2 and HePS-3 show a distribution of chain lengths as evidenced by the width of the peaks observed in size exclusion chromatography (Figure S1). Furthermore the observed compact sizes in solution by SAXS indicate that the HePSs are relatively flexible chains. For a completely flexible chain in dilute solution only one length scale is expected, namely the size of the random coil. The fact that we need two length-scales, $R_{\mathrm{G}}{ }^{2}$ and $R_{\mathrm{XS}}{ }^{2}$, to describe the elliptical cylinder chain conformation indicates limited flexibility in these HePSs similar to a description by the worm-like-chain model. ${ }^{51,52}$ 
Constrained modeling of HePS-1. Compared to the linear HePS-1 model, the experimental $R_{\mathrm{G}}$, $R_{\mathrm{XS}}$ and maximum length $L$ values showed that HePS-1 is not extended but exhibits a coiled conformation in solution. Here we used a constrained scattering modeling approach to provide molecular explanation for these scattering data. Modeling for HePS-1 was performed using the extended model created using its molar mass and repeat structure as a starting constraint. In the models, the glycosidic linkage connectivity between oligosaccharide rings was maintained, while the

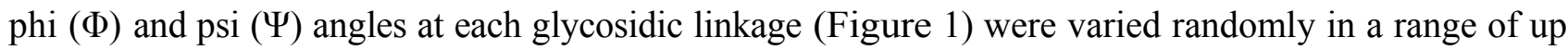
to $\pm 45^{\circ}$ from their starting values. A total of 15,000 conformational models for HePS- 1 were generated. Scattering curves for these randomized models were calculated and fitted to the experimental curves and $R_{\mathrm{G}}, R_{\mathrm{XS}}$ and $R$ factors were calculated for all modeled curves. The $R$ factor distributions (Figure 3) showed that all 15,000 HePS-1 randomized models (filled circles) encompassed the experimental $R_{\mathrm{G}}$ and $R_{\mathrm{XS}}$ values (dashed lines) and that the best $R$ factor values were well below the level of $5 \%$ that is expected for excellent scattering curve fittings. ${ }^{28}$ This showed that 15,000 randomized models provided sufficient conformers to be able to determine the best-fit HePS-1 solution structures. The lowest $R$ factor had $R_{\mathrm{G}}$ and $R_{\mathrm{XS}}$ values (red and blue circles) that agree well with the experimental $R_{\mathrm{G}}$ and $R_{\mathrm{XS}}$ values.

The modeling analysis of HePS-1 showed good agreement between models and experimental scattering data. A family of five best-fit HePS-1 models were selected based on the $R$ factor, $R_{\mathrm{G}}$ and $R_{\mathrm{XS}}$ parameters. All five best-fit models gave $R$ factor of $3.9 \%$ to $4.6 \%, R_{\mathrm{G}}$ values of 9.90-10.84 nm, $R_{\mathrm{Xs}}$ values of 3.14-3.47 $\mathrm{nm}$ and maximum length $L$ of $42-44 \mathrm{~nm}$ (Table 1). All these agreed well with the corresponding experimental values of $10.14 \pm 0.31 \mathrm{~nm}, 3.22 \pm 0.09 \mathrm{~nm}$, and $41.0 \mathrm{~nm}$. In contrast, the linear HePS-1 model gave a much poorer fit with an $R$ factor of $29.9 \%, R_{\mathrm{G}}$ of 


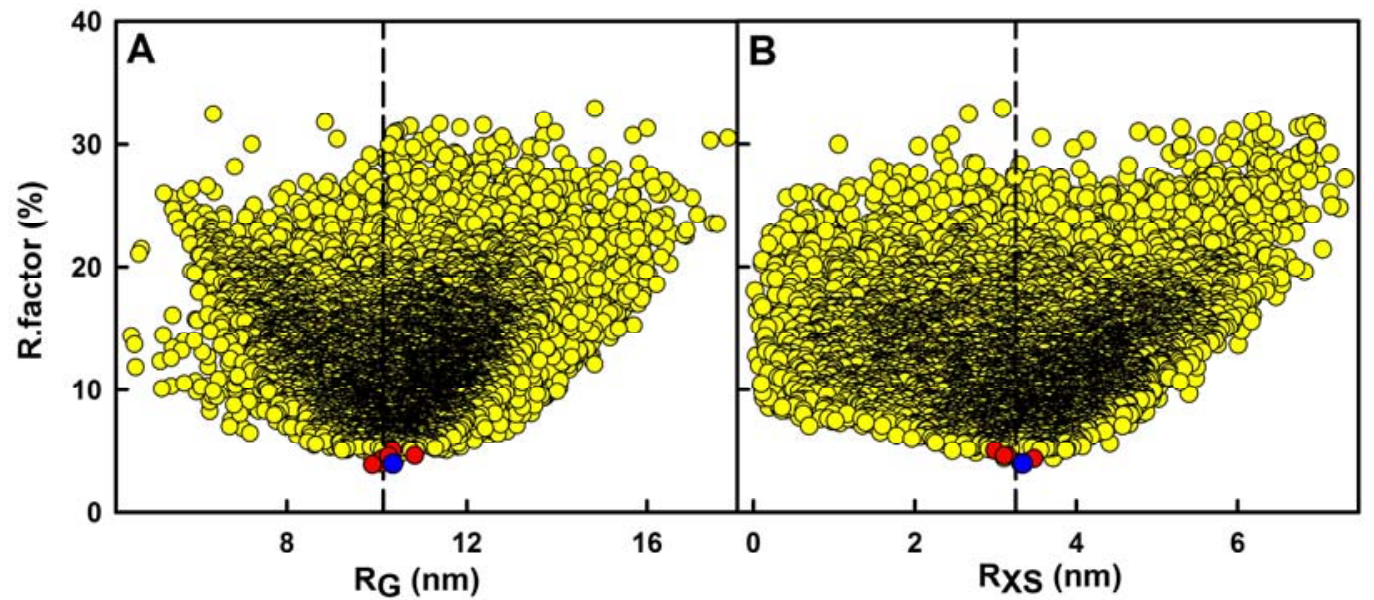

Figure 3. Constrained modelling analyses of HePS-1. (A) The $R$ factor values for the 15,000 conformational models of HePS-1 are compared with their $R_{\mathrm{G}}$ values. The vertical broken line corresponds to the experimental $R_{\mathrm{G}}$ value. The blue circle represents the best-fit model and the other four best-fit models are shown in red close to the $R$ factor minimum. (B) The $R$ factor values for the 15,000 conformational models of HePS-1 are compared with their $R_{\mathrm{XS}}$ values. The blue circle represents the best-fit model and the four other best-fit models are shown in red close to the $R$ factor minimum.

$91 \mathrm{~nm}, R_{\mathrm{XS}}$ of $1.1 \mathrm{~nm}$ and $L$ of $321 \mathrm{~nm}$. Modeling curve fitting analysis showed excellent visual agreement between the experimental $I(Q)$ curve and the modeled $I(Q)$ curve, and between the experimental $P(r)$ curve and the modeled $P(r)$ curve (Figure 4A). In conclusion, the modeling analyses for the best-fit HePS-1 models show coil-like structures in solution as may be seen in Figures 4B and D. 

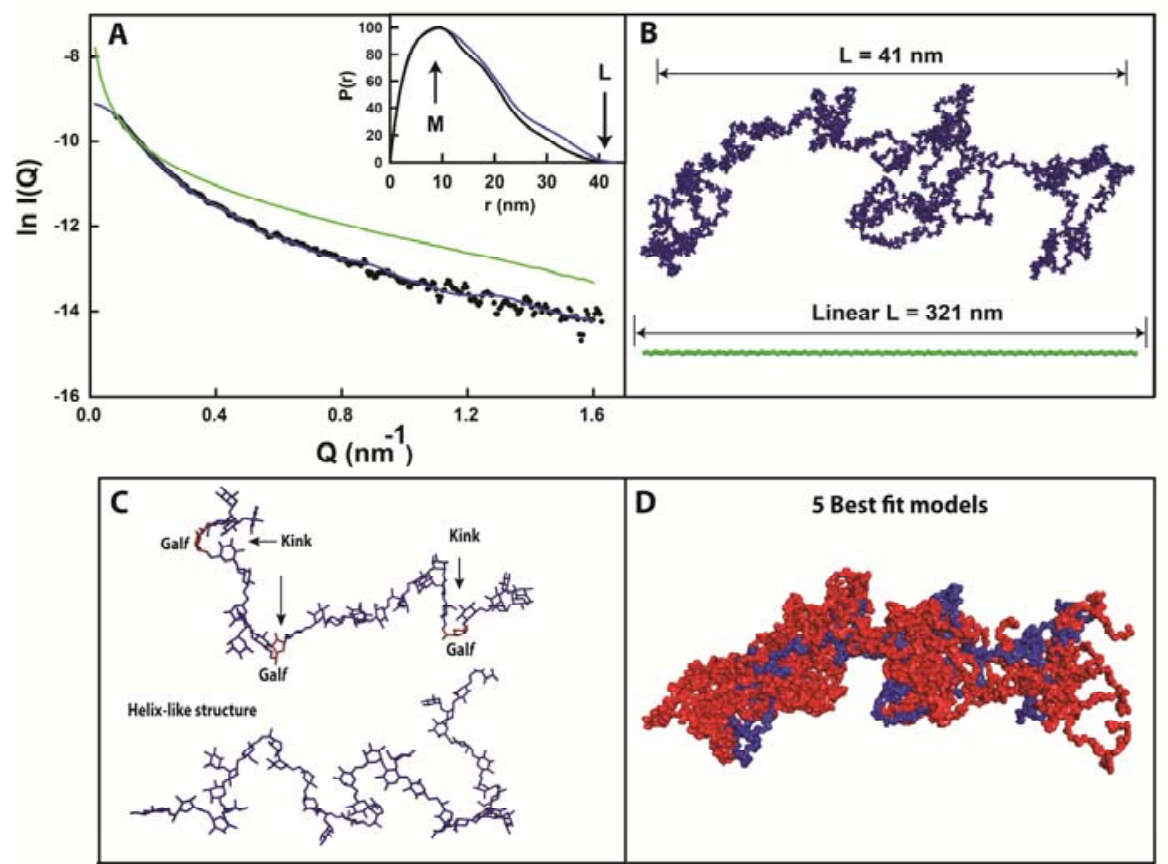

Figure 4. X-ray constrained scattering modeling curve fits for best-fit and linear-fit HePS-1 models. The data in "A" depicts the $I(Q)$ curves, and the inset shows the $P(r)$ distance distribution functions. The experimental $I(Q)$ and $P(r)$ scattering data are shown by black circles or lines, respectively; the blue line and model correspond to the best-fit HePS-1 model selected from 15,000 conformational models. The green line and model correspond to the linear poor-fit HePS-1 model. The maximum lengths $L$ of best-fit model (blue) is shown in the inset for comparison with their $L$ values in the $P(r)$ curves. (B) The best-fit and linear models are shown in blue and green, respectively (length scales are different). (C) Oligosaccharide fragments generated from the best-fit model in B; the top one shows kink structures that produced by Galf, the bottom one shows a helix-like structure. (D) Superimposition of the five best-fit models for HePS-1. The five best-fit HePS-1 models were superimposed using Discovery Studio VISUALISER software. The best-fit model from Fig. 3 is shown in blue, while the related best-fit models are shown in red.

Conformation of HePS-1. For large, intact HePS, it is not possible to generate conformationally randomized models and look into their flexibility using NMR and other techniques. Using a constrained scattering modelling approach, we were able to generate conformational models and derive structural parameters. In terms of the glycosidic linkages, information on conformation in the HePS structures was obtained from analyses of the $\Phi$ and $\Psi$ 
angles. The HePSs with stiff glycosidic linkages tend to occupy larger volume in solution compared to flexible linkages, and hence contribute significantly to increase viscosity. The $\beta$ $(1 \rightarrow 4)$ linkages are stiffer than the $\beta-(1 \rightarrow 3)$ and $\beta-(1 \rightarrow 2)$ glycosidic linkages, while the $\alpha$-linkages $(1 \rightarrow 2,1 \rightarrow 3$ and $1 \rightarrow 6)$ are flexible. ${ }^{21,} 27$ Therefore HePSs containing more $\alpha$-linkages in the oligosaccharide repeat units are in general more flexible.

The average $\Phi$ and $\Psi$ angles of the HeSP-1 $\alpha$-D-Gal $p$-( $1 \rightarrow 3)-\beta$-L-Rhap glycosidic bonds were $76^{\circ}$ and $94^{\circ}(260$ values) and $\beta$-L-Rhap-( $1 \rightarrow 4)-\beta$-D-Glc $p$ were $58^{\circ}$ and $111^{\circ}$ (260 values) respectively (Table 2$)$. For $\beta-(1 \rightarrow 6)$ linkages (four linkages in total), the average $\Phi$ angles ranged from $-86^{\circ}$ to $161^{\circ}$ (each 260 values) and the $\Psi$ angles ranged from $-174^{\circ}$ to $159^{\circ}$ (each 260 values) (Table 2). Here the standard deviation of $\Phi$ angles have increased to $33-43^{\circ}$, while the standard deviation of $\Psi$ angles were similar at $40^{\circ}$. Since the repeating units in HePS-1 contain three $(1 \rightarrow 6$ )-linkages (one $\alpha$ and two $\beta$ ), of which two are consecutive with a five-membered ring (Galf) which makes HePS-1 more flexible, a very large number of short- and long-range orientations in the main chain are possible. This could be explained by $\Phi$ and $\Psi$ angles analyses and increase in standard deviation of $(1 \rightarrow 6)$ linkages with five-membered ring (Galf), indicating that fivemembered ring Galf residues likely produce kinks or bents in the HePS chain thus showing higher conformational flexibility (Figure 5). Due to presence of these Galf residues in repeating units which tend to produce progressive bending or kinks, the HePS-1 chain possesses secondary structural elements that potentially adopt random coil-like structures in solution (Figure 4B and C). Modeling and simulation studies (no experimental SAXS data) have also shown that the HePSs form secondary structural elements, and that the Galf has a significant influence on the conformational properties of HePSs. ${ }^{53,54}$ SAXS studies of HePS (unknown size) from marine 
bacterium at $0.4 \%(\mathrm{w} / \mathrm{v}$, i.e. $4 \mathrm{mg} / \mathrm{ml})$ have shown that HePS forms a network of random coiled polymeric chains that was demonstrated to be relatively homogeneous at high $\mathrm{pH}$ and less homogeneous at very low $\mathrm{pH}^{55}$ Using the SAXS technique, Stokke et al. have reported that alginate (anionic polysaccharides) forms a network of heterogeneous gels depending on the alginate concentration, $\mathrm{Ca}^{2+}$ concentration and its composition. ${ }^{56}$ The solution-state behaviors of EPSs differ substantially in their 3D structures, degree of branching, conformational flexibility of backbone, charge and their ability to interact with proteins. ${ }^{22-25}$ Besides these factors, conformational flexibilities of monosaccharides and the nature of glycosidic bonds in their repeat units also greatly contribute to solution-state behavior of EPSs. ${ }^{10,57}$ Based on the $\Phi$ and $\Psi$ angles analyses derived from best-fit HePS-1 structure, we showed that HePS-1 has significant flexibility due to presence of Gal $f$ residues and three $(1 \rightarrow 6)$-linkages in repeating units, and in dilute solution at $1 \mathrm{mg} / \mathrm{ml}$ it behaves as a random coil-like structure. The superimpositions showed that the five best-fit structures exhibited helix-like and random coil-like structures (Figure 4B and D). In their natural environment, the structural flexibility and the coil-like structures of HePSs may protect the bacteria against environmental stress. In proteinfree system (in vitro), we speculate that the coil-like structure may not contribute significantly to viscosity because it may occupy less volume compared to its extended chain conformation. In proteincontaining system, the coil-like structure may stabilize the system by forming optimal surface contacts with proteins, and thus it can be used for various applications. 
Table 2. Summary of $\Phi$ and $\Psi$ angles in HePS-1 structures

\begin{tabular}{|c|c|c|}
\hline Glycosidic Linkage & $\begin{array}{l}\mathrm{O}_{5}-\mathrm{C}_{1}-\mathrm{O}_{1}-\mathrm{C}^{\prime}{ }_{\mathrm{X}} \\
\Phi\left(^{\circ}\right)\end{array}$ & $\begin{array}{l}\mathrm{C}_{1}-\mathrm{O}_{1}-\mathrm{C}^{\prime}{ }_{\mathrm{X}}-\mathrm{C}^{\prime}{ }_{\mathrm{X}-1} \\
\Psi\left(^{\circ}\right)\end{array}$ \\
\hline \multirow[t]{2}{*}{$\alpha-\mathrm{D}-\mathrm{Gal} p-(1 \rightarrow 3)-\beta-\mathrm{L}-\mathrm{Rha} p$} & $76 \pm 29$ & $94 \pm 33$ \\
\hline & 76 & 93 \\
\hline \multirow[t]{2}{*}{$\beta$-L-Rha $p-(1 \rightarrow 4)-\beta$-D-Glc $p$} & $58 \pm 40$ & $111 \pm 33$ \\
\hline & 66 & 110 \\
\hline \multirow[t]{2}{*}{$\beta$-D-Glc $p-(1 \rightarrow 6)-\beta$-D-Gal $p$} & $-77 \pm 33$ & $-160 \pm 40$ \\
\hline & -71 & -160 \\
\hline \multirow[t]{2}{*}{$\beta$-D-Gal $p-(1 \rightarrow 6)-\alpha-\mathrm{D}-\mathrm{Gal} p$} & $-86 \pm 42$ & $-149 \pm 40$ \\
\hline & -75 & -160 \\
\hline \multirow[t]{2}{*}{$\beta$-D-Glc $p-(1 \rightarrow 6)-\alpha$-D-Gal $f$} & $-74 \pm 43$ & $-174 \pm 40$ \\
\hline & -73 & -160 \\
\hline \multirow[t]{2}{*}{$\alpha$-D-Gal $f-(1 \rightarrow 6)-\beta-\mathrm{D}-\mathrm{Glc} p$} & $161 \pm 43$ & $-159 \pm 40$ \\
\hline & 175 & -160 \\
\hline
\end{tabular}

The first $\Phi$ and $\Psi$ values are from the best-fit HePS-1 models, and the second values are from the starting HePS-1 model (Materials and Methods). 


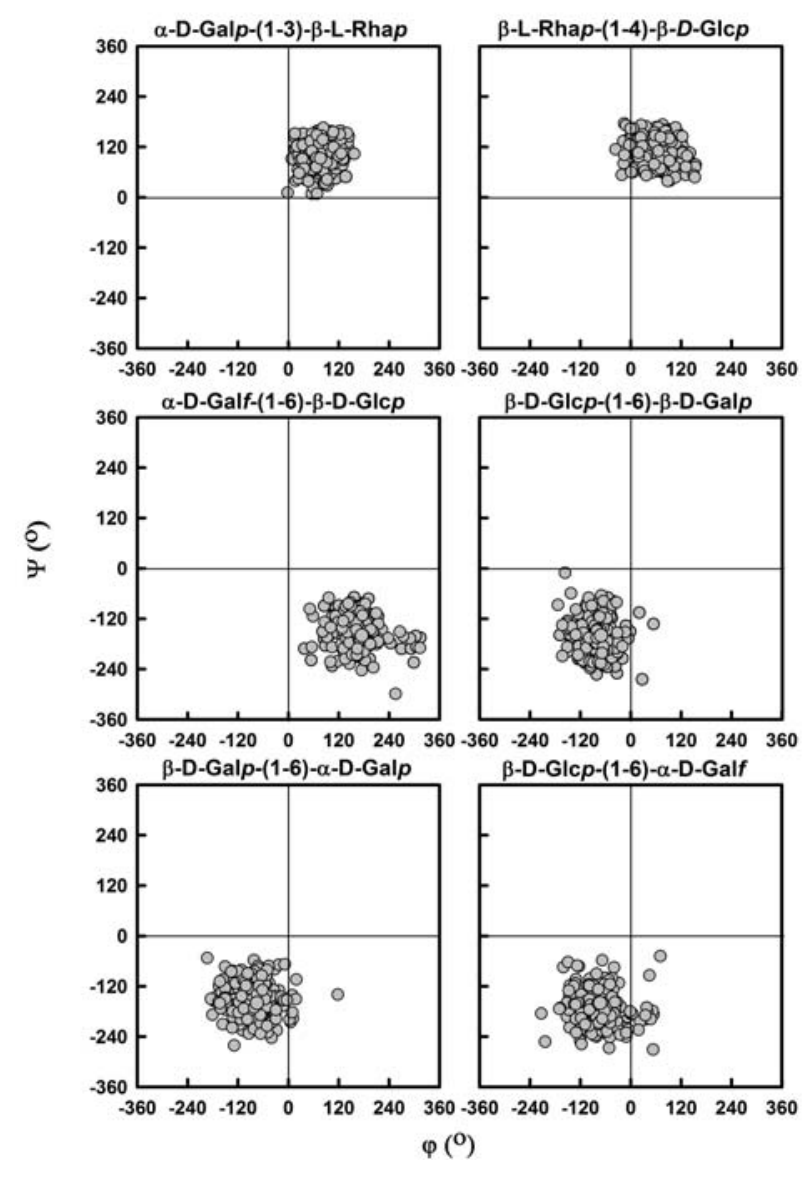

Figure 5. Plots of glycosidic dihedral angles for the HePS-1. Phi $(\Phi)$ and Psi $(\Psi)$ angles, in all cases, are shown by gray circles. The dihedral angles of glycosidic linkages of disaccharides $(1 \rightarrow 3)$ are defined as $\Phi=\mathrm{O}^{\circ}-\mathrm{C} 1{ }^{\circ}-\mathrm{O} 1-\mathrm{C} 3$ and $\Psi=\mathrm{C} 1^{\circ}-\mathrm{O} 1-\mathrm{C} 3-\mathrm{C} 2$. For glycosidic linkages $(1 \rightarrow 4)$, the atoms defining $\Phi$ are $\mathrm{O}^{\circ}-\mathrm{C} 1^{\circ}-\mathrm{O} 1-\mathrm{C} 4$ and $\Psi$ are $\mathrm{C} 1^{\circ}-\mathrm{O} 1-\mathrm{C} 4-\mathrm{C} 3$. For glycosidic linkages $(1 \rightarrow 6)$, the atoms defining $\Phi$ are $\mathrm{O}^{\circ}-\mathrm{C} 1^{\circ}-\mathrm{O} 1-\mathrm{C} 6$ and $\Psi$ are $\mathrm{C} 1^{\circ}-\mathrm{O} 1-\mathrm{C} 6-\mathrm{C} 5$.

$A b$ initio scattering modeling of HePS-2 and HePS-3. Constrained scattering modeling was not done for HePS-2 and HePS-3 because of their large molecular size which hampered the generation of sufficient conformational models to be able to determine the best-fit 
models. Instead ab initio models for HePS-2 and HePS-3 were generated from their X-ray scattering curves, and in order to present overall shape envelopes for both of these HePSs in solution.

For HePS-2, ab initio scattering modeling analyses showed good agreements with SAXS data. The 20 HePS- 2 models gave an average $\operatorname{chi}^{2}\left(\chi^{2}\right)$ of $0.46 \pm 0.02$ (where $\chi^{2}$ is the discrepancy between theoretical and experimental curves) and the best-fit model gave $\chi^{2}$ of 0.43 . The best-fit model gave $R_{\mathrm{G}}$ of $11.56 \mathrm{~nm}$ and $L$ of $39.6 \mathrm{~nm}$, in good agreement with the experimental GNOM $R_{\mathrm{G}}$ of $11.49 \pm 0.25$ $\mathrm{nm}$ and $L$ of $38.0 \mathrm{~nm}$ (Table 1). The $P(r)$ curve for the best-fit model and their $r$ values agree with experimental data (Figure 6, inset). The visual agreement between the experimental $I(Q)$ curve and the modeled $I(Q)$ curve was excellent (Figure 6).

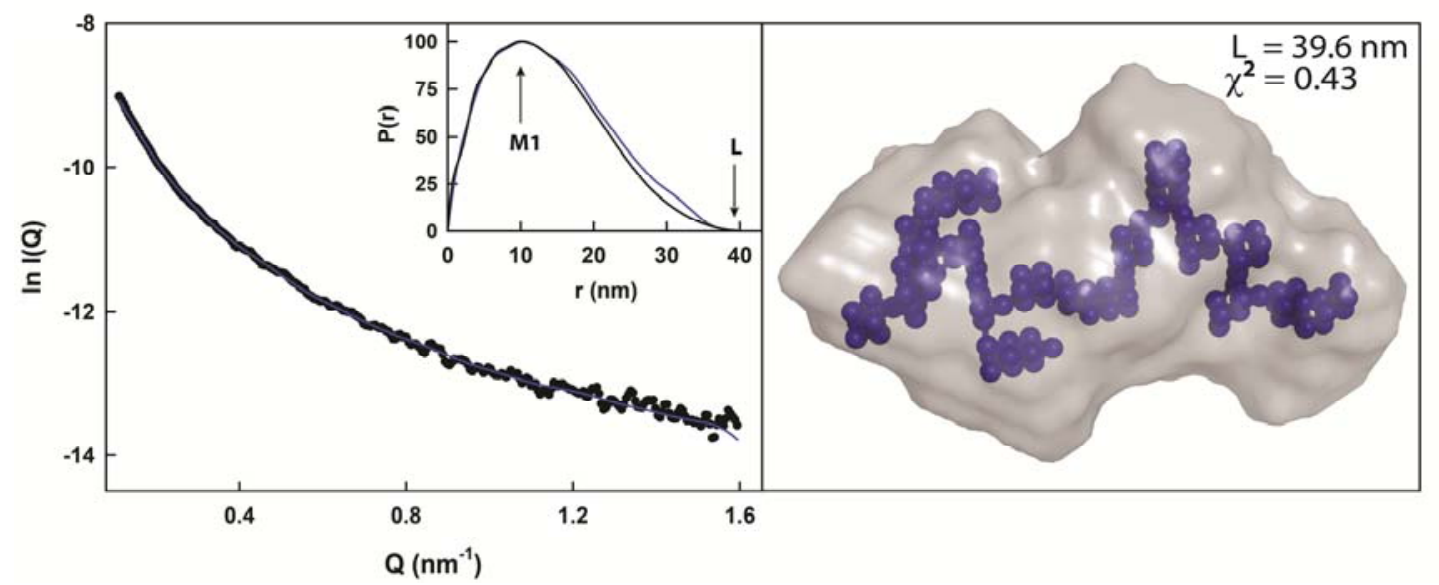

Figure 6. The $a b$ initio solution scattering curve fit for the best-fit HePS-2 model. The data on the left are the modeled scattering $I(Q)$ curve for the best-fit ab initio HePS-2 model (blue line) fitted to the experimental scattering data (black circles). The best-fit (blue spheres) and the average of 20 models indicated by light gray surface are shown to the right. The maximum lengths $L$ of best-fit ab initio model (blue) and experimental $L$ are compared in the $P(r)$ curve (inset).

For HePS-3, ab initio modeling analyses showed similar good agreements with the experimental SAXS data. The 20 HePS- 3 models generated from its X-ray scattering data gave an average $\chi^{2}$ of 0.37 \pm 0.01 and the best-fit model gave $\chi^{2}$ value of 0.34 . The best-fit modeled curve $I(Q)$ showed excellent 
visual agreement with the experimental $I(Q)$ (Figure 7). The best-fit model gave $R_{\mathrm{G}}$ of $11.63 \mathrm{~nm}$ and maximum length $L$ of $39 \mathrm{~nm}$, in good agreement with the experimental $R_{\mathrm{G}}$ of $11.44 \pm 0.1 \mathrm{~nm}$ and $L$ of $41.8 \mathrm{~nm}$ (Table 1). The $P(r)$ curve for the best-fit model and their $r$ values agree well with those seen experimentally (Figure 7, inset). In conclusion, the best-fit ab initio scattering models for both HePS-2 and HePS-3 also show compact structures.

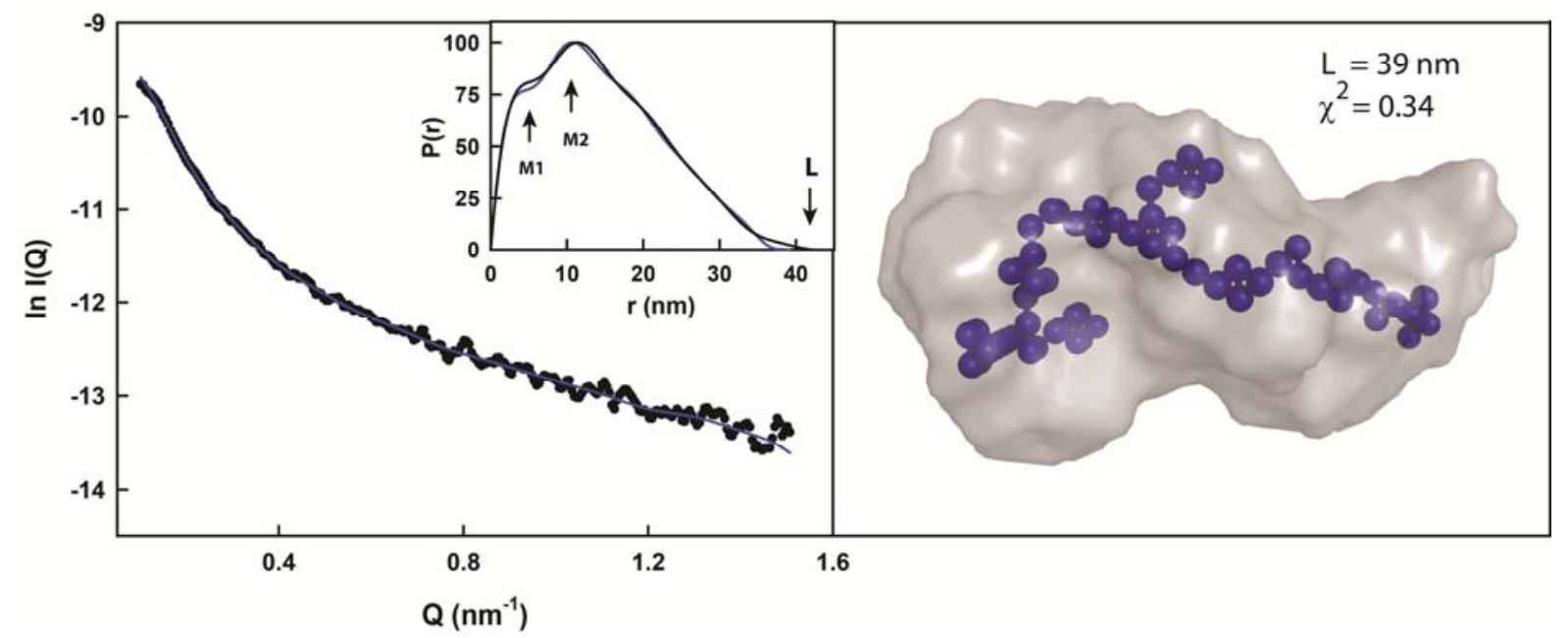

Figure 7. The ab initio X-ray scattering curve fit for the best-fit HePS-3 model. The data on the left are the modeled scattering $I(Q)$ curve for the best-fit ab initio HePS-3 model (blue line) fitted to the experimental scattering data (black circles). The best-fit (blue spheres) and the average of 20 models indicated by light gray surface are shown to the right. The maximum lengths $L$ of best-fit ab initio model (blue) and experimental $L$ are compared in the $P(r)$ curve (inset).

Dynamic light scattering (DLS) of HePS-1, HePS-2 and HePS-3. DLS assesses the size of macromolecules in solution by following their diffusion rates under Brownian motion. In DLS experiments, sizes of the 
three HePSs $(0.5-1 \mathrm{mg} / \mathrm{mL})$ in $10 \mathrm{mM}$ sodium citrate buffer $\mathrm{pH} 4.0$ were analyzed by using particle sizing software. HePS-1 gave a single peak with average hydrodynamic diameter $\left(d_{\mathrm{H}}\right)$ of $29.9 \pm 0.2$ $\mathrm{nm}$ (Figure 8A), which is smaller than the maximum length $L$ determined by SAXS (Table 1). The $d_{\mathrm{H}}$

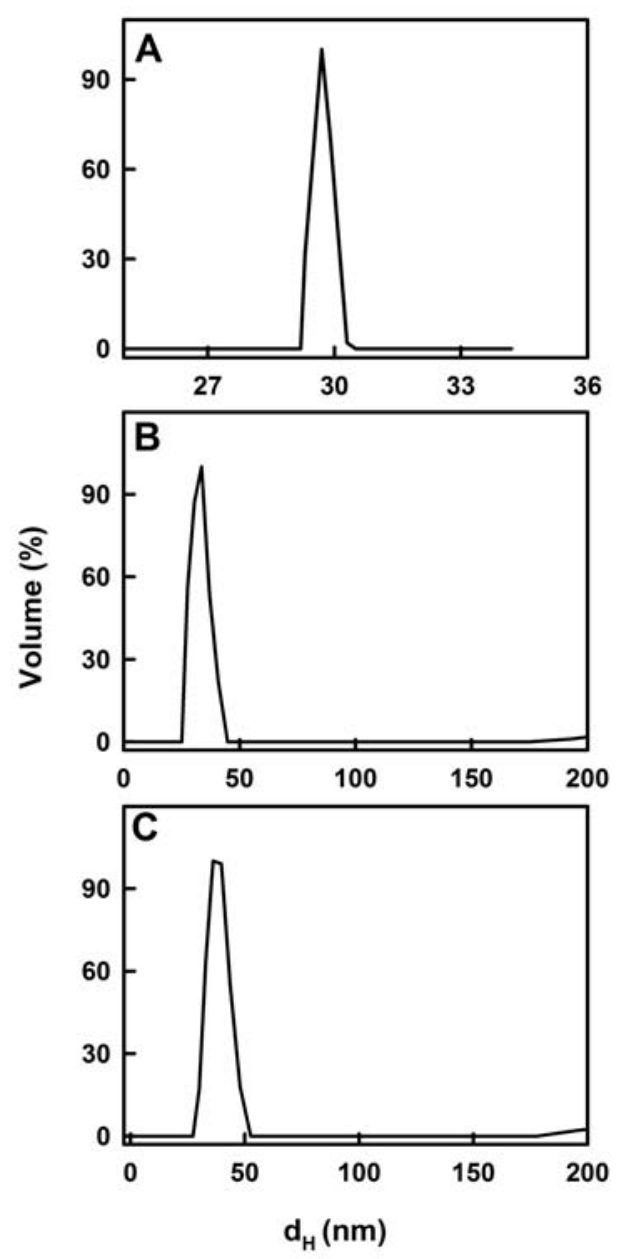

Figure 8. Dynamic light scattering data analyses of HePS-1. HePS-2 and HePS-3. Hydrodynamic diameter $\left(d_{\mathrm{H}}\right)$ values of $29.7 \mathrm{~nm}$ for HePS-1 (A), $33.6 \mathrm{~nm}$ for HePS-2 (B) and $36.1 \mathrm{~nm}$ for HePS-3 (C). The DLS experiments were performed in $10 \mathrm{mM}$ citrate buffer $\mathrm{pH} 4.0$.

of HePS-2 and HePS-3 were determined to be $34.1 \pm 0.3$ and $35.6 \pm 0.5 \mathrm{~nm}$, respectively (Figure $8 \mathrm{~B}$ and C). In both Figure 8B and C a minor peak appeared at high $d_{\mathrm{H}}$ values of 240-260 nm which 
could be attributable to dust particles (not shown). These $d_{\mathrm{H}}$ values of HePS-2 and HePS-3 also show slight deviation from their scattering lengths (Table 1), and reflect the difference in accuracy of the two techniques. In DLS, $d_{\mathrm{H}}$ is the diameter of the hard sphere that diffuses at the same rate as the molecule being measured. Like X-ray data, these $d_{\mathrm{H}}$ values are also much smaller than those measured for the extended HePS-1, HePS-2 and HePS-3 models, thus supporting the X-ray scattering data.

\section{CONCLUSIONS}

Knowledge on molecular structures of HePSs is essential to understand their function and the relationship between their structure, and their physical and rheological properties. Despite information on primary structures of oligosaccharide repeats as determined by NMR spectrometry, molecular structures for full-length, intact HePSs have up to now not been reported. In the present study, we used SAXS and DLS in combination with constrained scattering modeling to characterize molecular structures of three LAB-HePSs in solution. SAXS data show that the experimental $R_{\mathrm{G}}, R_{\mathrm{Xs}}$ and maximum lengths $L$ values of HePS-1, HePS-2 and HePS-3 do not show linear relationship with the corresponding modeled values calculated for their extended chain lengths, suggesting that these HePSs exhibit compact structures of random coil-like conformation in solution. Here a constrained modeling approach previously used to determine molecular structures for multi-domain proteins and more recently for anionic oligosaccharides, ${ }^{31-34}$ was extended to study full-length, large HePS. Constrained scattering modeling determines a 3D medium-resolution structure and represents a family of best-fit structures that best accounts for the experimental scattering data, suited also to derive structural information on conformational flexibility of HePSs. Constrained scattering modeling of HePS-1 
validated the experimental scattering data analysis. From analyses of $\Phi$ and $\Psi$ angles of the best-fit models, the findings suggest that consecutive $(1 \rightarrow 6)$-linkages with Gal $f$ residues are more flexible and produce progressive bents in the backbone tending to adopt non-uniform local helical and random coillike structures.

Ab initio scattering modeling analyses showed that HePS-2 and HePS-3 also adopt a coil-like conformation in solution. Although these HePSs differ in their molecular sizes, their maximum length values in solution were very close to each other, showing that the high molecular weight HePS has more compact conformation than the smaller one, and this could be explained by high degree of branching and flexibility of the backbone. The bends in the HePS-1 structures arise through kinks produced by Gal $f$ in its chain. In the case of anionic polysaccharides interactions with proteins, it has already been established that kink structure provided optimal electrostatic and hydrogen bond contacts with proteins. ${ }^{58}$ In terms of interactions of HePSs with proteins, the bent structures may facilitate optimal contact formation between HePSs and proteins, and may form stable complexes.

\section{ASSOCIATED CONTENT}

\section{Supporting information}

Size exclusion chromatography of HePS-1, HePS-2 and HePS-3

\section{AUTHOR INFORMATION}

\section{Corresponding Author}

Department of Micro- and Nanotechnology, DTU, Ørsteds Plads, building 423, DK-2800 Kgs. Lyngby, Denmark. phone:+4545258144.*Email:kral@nanotech.dtu.dk.

\section{ACKNOWLEDGEMENTS}

We thank Karina Jansen and Lotte Nielsen for their valuable technical assistance. This project was funded by the Danish Research Council for Independent Research | Technical and Production Sciences 
to the project "HEXPIN. Associative interactions between exopolysaccharides from lactic acid baceria and milk proteins. Gaining insights deployable in design and optimised food texture". We also thank Prof. Luc de Vuyst, Vrije Universiteit of Brussel, Belgium, for providing the Streptococcus thermophilus EU20 strain. The MAXIV synchrotron and the I911-SAXS beamline crew are acknowledged for beam-time and support during data-collection. The research presented has received funding from BioStruct-X and DANSCATT (the Danish agency and for Science, Technology and Innovation).

\section{REFERENCES}

(1) Donot, F.; Fontana, A.; Baccou, J. C.; Schorr-Galindo, S. Carbohydr. Polym. 2012, 87, 951-962.

(2) Moscovici, M. Front. Microbiol. 2015, 6, 1-11.

(3) Dertli, E.; Colquhoun, I. J.; Gunning, A. P.; Bongaerts, R. J.; Le Gall, G.; Bonev, B. B.; Mayer, M. J.; Narbad, A. J. Biol. Chem. 2013, 288, 31938-31951.

(4) Laws, A.; Gu, Y.; Marshall, V. Biotechnol. Adv. 2001, 19, 597-625.

(5) Torino, M. I.; Font de Valdez, G.; Mozzi, F. Front. Microbiol. 2015, 6, 834..

(6) Ganzle, M.; Michael, G.; Schwab, C. Exopolysaccharide production by intestinal lactobacilli. In: Probiotics and prebiotics: Scientific aspects. Tannock, G. W (eds.). Norfolk, Caister Academic Press, 2005; pp 83-96.

(7) Schmid, J.; Sieber, V.; Rehm, B. Front. in Microbiol. 2015, 6, 1-24. 
(8) Górska-Frączek, S.; Sandström, C.; Kenne, L.; Rybka, J.; Strus, M.; Heczko, P.; Gamian, A. Carbohydr. Res. 2011, 346, 2926-2932.

(9) Ruas-Madiedo, P.; de los Reyes-Gavilán, C. G. J. Dairy Sci. 2005, 88, 843-856.

(10) Caggianiello, G.; Kleerebezem, M.; Spano, G. Appl. Microbiol. Biotechnol. 2016, 100, 3877-86.

(11) Badel, S.; Bernardi, T.; Michaud, P. Biotechnol. Adv. 2011, 29, 54-66.

(12) Ravishankar Rai V, Jamuna A. Bai (2014). Beneficial Microbes in Fermented and Functional Foods. December 17, by CRC Press Reference - 600 Pages - 93 B

(13) Patel, A.; Prajapati, J. B. Adv. Dairy Res. 2013, 1, 1-7.

(14) Levrat-Verny, M. A.; Behr, S.; Mustad, V.; Rémésy, C.; Demigné, C. J. Nutr. 2000, 130, 243248.

(15) Maeda, H.; Zhu, X.; Suzuki, S.; Suzuki, K.; Kitamura, S. J. Agric. Food Chem. 2004, 52, 55335538.

(16) Kim, Y.; Oh, S.; Kim, S. H. Biochem. Biophys. Res. Commun. 2009, 379, 324-329.

(17) Gentès, M-C.; St-Gelais, D.; Turgeon, S. L. Dairy Sci. Technol. 2013, 93, 255-271.

(18) Salazar, N.; Gueimonde, M.; de Los Reyes-Gavilán, C. G.; Ruas-Madiedo, P. Crit. Rev. Food Sci. Nutr. 2016, 56,1440-1453.

(19) Doleyres, Y. L.; Schaub, L.; Lacroix, C. J. Dairy Sci. 2005, 88, 4146-4156.

(20) Ruas-Madiedo, P.; Tuinier, R.; Kanning, M.; Zoon, P. Int. Dairy J. 2002, 12, 689-695. 
(21) Faber, E. J.; Zoon, P.; Kamerling, J. P.; Vliegenthart, J. F. G. Carbohydr. Res. 1998, 310, 269276.

(22) Tuinier, R.; Ten Grotenhuis, E.; Holt, C.; Timmins, P. A.; De Kruif, C. G. Phys. Rev. E 1999, $60,848-856$.

(23) Looijesteijn, P. J.; Schols, H. A.; Voragen, A. G. J.; Zoon, P. Biopolymers 2001, 59, 160-166.

(24) Girard, M.; Schaffer-Lequart, C. Int. Dairy J. 2007, 17, 666-673.

(25) Turgeon, S. L.; Plesca, V. Proceedings of the 5th International Symposium on Food Rheology and Structure, Zurich, Switzerland, 2009; pp 416-419.

(26) Whistler, R. L., BeMiller, J. N. Carbohydrate chemistry for food scientists. Eagan Press, St Paul, Minnesota, 1997; pp 117-151.

(27) Tuinier, R.; Zoon, P.; Cohen-Stuart, M. A.; Fleer, G. J.; de Kruif, C. G. Biopolymers 1999, 50, $641-646$.

(28) Marshall, V. M.; Dunn, H.; Elvin, M.; McLay, N.; Gu, Y.; Laws, A. P. Carbohydr. Res. 2001b, $331,413-422$.

(29) Landersjo, C.; Yang, Z.; Huttunen, E.; Widmalm, G. Biomacromolecules 2002, 3, 880-884.

(30) Goh, K. K.; Haisman, D. R.; Singh, H. Appl. Microbiol. Biotechnol. 2005, 67, 202-208.

(31) Perkins, S. J.; Okemefuna, A. I.; Fernando, A. N.; Bonner, A.; Gilbert, H. E.; Furtado, P. B. Methods Cell Biol. 2008, 84, 375-423. 
(32) Perkins, S. J.; Okemefuna, A. I.; Nan, R.; Li, K.; Bonner, A. J. R. Soc., Interface 2009, 6, S679S696.

(33) Khan, S.; Fung, K. W.; Rodriguez, E.; Patel, R.; Gor, J.; Mulloy, B.; Perkins, S. J. J. Biol. Chem. 2013, 288, 27737-27751.

(34) Khan, S.; Gor, J.; Mulloy, B.; Perkins, S. J. J. Mol. Biol. 2010, 395, 504-521.

(35) Gruter, M.; Leeflang, B. R.; Kuiper, J.; Kamerling, J. P.; Vliegenthart, J. F. Carbohydr. Res. 1993, 239, 209-226.

(36) DuBois, M.; Gilles, K. A.; Hamilton, J. K.; Rebers, P. A.; Smith, F. Anal. Chem. 1956, 28, 350356.

(37) Labrador, A.; Cerenius, Y.; Svensson, C.; Theodor, K.; Plivelic, T. J. Phys.: Conf. Ser. 2013, 425, 072019.

(38) Petoukhov, M. V.; Franke, D.; Shkumatov, A. V.; Tria, G.; Kikhney, A. G.; Gajda, M.; Gorba, C.; Mertens, H. D. T.; Konarev, P. V.; Svergun, D. I. J. Appl. Crystallogr. 2012, 45, 342-350.

(39) Glatter, O.; Kratky, O. (Editors): Small-Angle X-ray Scattering. Academic Press, New York, NY, 1982.

(40) Semenyuk, A. V.; Svergun, D. I. J. Appl. Crystallogr. 1991, 24, 537-540.

(41) Bohne,A.; Lang, E.; von der Lieth, C. W. J. Mol. Model. 1998, 4, 33-43.

(42) Bohne,A.; Lang, E.; von der Lieth, C.W. Bioinformatics 1999, 15, 767-768

(43) Mayo, S. L.; Olafson, B. D.; Goddard, W. A. J. Phys. Chem. 1990, 94, 8897-8909. 
(44) Wright, D. W.; Perkins, S. J. J. Appl. Crystallogr. 2015, 48, 953-961

(45) Okemefuna, A. I.; Nan, R.; Gor, J.; Perkins, S. J. J. Mol. Biol. 2009, 391, 98-118.

(46) Ashton, A. W.; Boehm, M. K.; Gallimore, J. R.; Pepys, M. B.; Perkins, S. J. J. Mol. Biol. 1997, $272,408-422$

(47) Perkins, S. J.; Weiss, H. J. Mol. Biol. 1983, 168, 847-866.

(48) Franke, D.; Svergun, D. I. J. Appl. Crystallogr. 2009, 42, 342-346.

(49) Volkov, V. V.; Svergun, D. I. J. Appl. Crystallogr. 2003, 36, 860-864.

(50) Petoukhov, M. V.; Svergun, D. I. Biophys. J. 2005, 89, 1237-1250.

(51) Li, Y.; Huang, Q.; Shi, T. J. Phys. Chem. B 2006, 110, 23502-23506.

(52) Cheng, G.; Melnichenko, Y. B.; Wignall, G. D.; Hua, F.; Hong, K.; Mays, J. W. Macromolecules 2008, 41, 9831-9836.

(53) Sánchez-Medina, I.; Frank, M.; von der Lieth, C. W.; Kamerling, J. P. Org. Biomol. Chem. 2009, 7, 280-287.

(54) Faber, E. J.; van Kuik, J. A.; Kamerling, J. P. Vliegenthart, J. F. Biopolymers 2002, 63, 66-76.

(55) Dogsa, I.; Kriechbaum, M.; Stopar, D.; Laggner, P.Structure of bacterial extracellular polymeric substances at different pH values as determined by SAXS. Biophys. J. 2005, 89, 2711-2720. 
(56) Stokke, B. T.; Draget, K. I.; Smidsrod, O.; Yuguchi, Y.; Urakawa, H.; Kajiwara, K. Macromolecules 2000, 33, 1853-1863. (57) Hallemeersch, I., De Baets, S., Vandamme, E. J. Biopolymers 2002, 5, 407-429.

(58) Raman, R., Venkataraman, G., Ernst, S., Sasisekharan, V.; Sasisekharan, R. Proc. Natl. Acad. Sci. U.S.A. 2003, 100, 2357-2362. 
Table of Contents graphic (TOC)

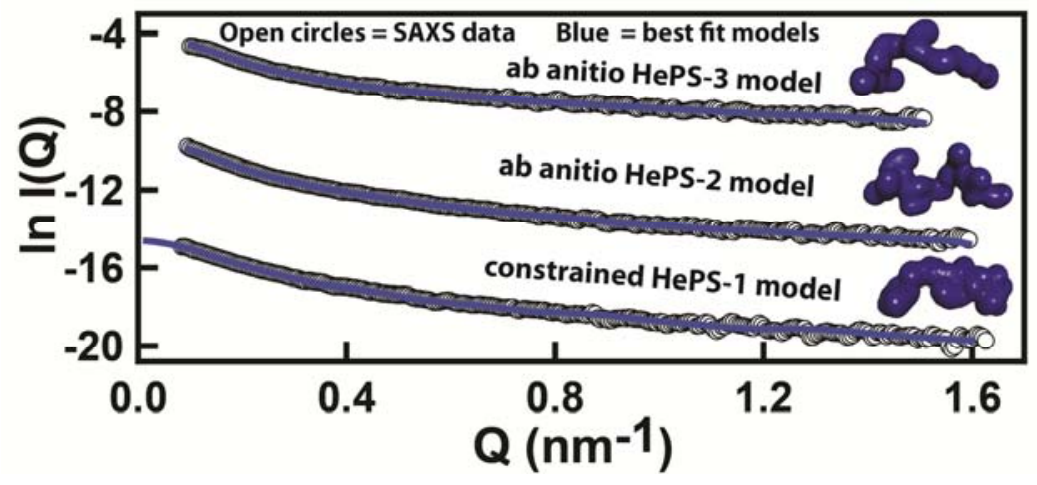

